\title{
Detection of the Binary Companion to the Progenitor of SN 1993J
}

\author{
S.J. Smartt ${ }^{1}$, J.R. Maund ${ }^{1}$, R.P. Kudritzki ${ }^{2}$, P. Podsiadlowski ${ }^{3}$, G. Gilmore ${ }^{1}$ \\ 1 IoA, University of Cambridge, Cambridge, UH; \\ sjs@ast.cam . ac . uk, jrm@ast . cam . ac. uk \\ 2 IfA, University of Hawaii, Honolulu, HI, USA; \\ kudQifa.hawaii.edu \\ 3 University of Oxford, Oxford, UK; \\ podsi@astro.ox.ac.uk
}

Summary. We present a detailed late-time photometric and spectroscopic study of SN1993J with HST/ACS and Keck LRIS-B. We find a clear signature of a hot star component in the spectra of SN1993J which cannot be explained by the surrounding faint blue stars. This is the first detection of the expected massive binary companion to the red supergiant progenitor and confirms that SN1993J did indeed arise in an interacting binary system.

\section{The Late-time Lightcurve of $1993 \mathrm{~J}$}

The progenitor site of SN1993J was studied in detail shortly after discovery of the SN $[1,3]$. A progenitor star was identified with a measured spectral energy distribution (SED) in $U B V R I$. The $V R I$ colors were consistent with a K-type supergiant, but the $U B$-band fluxes were too high. This suggested there was flux from either a hot binary companion star, or a tight unresolved host OB association. Models of the supernova evolution at the time suggested the double peak in the lightcurve and the spectral transformation to a SN Ib could be explained if the K-type supergiant had lost almost all of its hydrogen envelope to a massive main-sequence (or near main-sequence) companion $[6,7,9]$. This would elegantly explain both the anomalous progenitor SED and the SN evolution. Several authors suggested that this binary companion could become visible within a few years after the SN faded. In this contribution we present a summary of our search for the companion of the progenitor star.

Figure 1 shows the $U B$ lightcurves of SN1993J (and SN1987A for comparison) to illustrate how the SN has faded and when we might likely be able to identify a progenitor star. Although these are very different types of $\mathrm{SNe}$, we don't have such continuous and late time coverage for any other SNe so our comparison data are limited. One difference is startlingly clear, after about 400 days SN1987A fades rapidly whereas SN1993J seems to plateau out at absolute magnitudes of around -8 . Late-time low resolution spectra of 1993J (see the Filippenko \& Matheson contribution in these proceedings) 

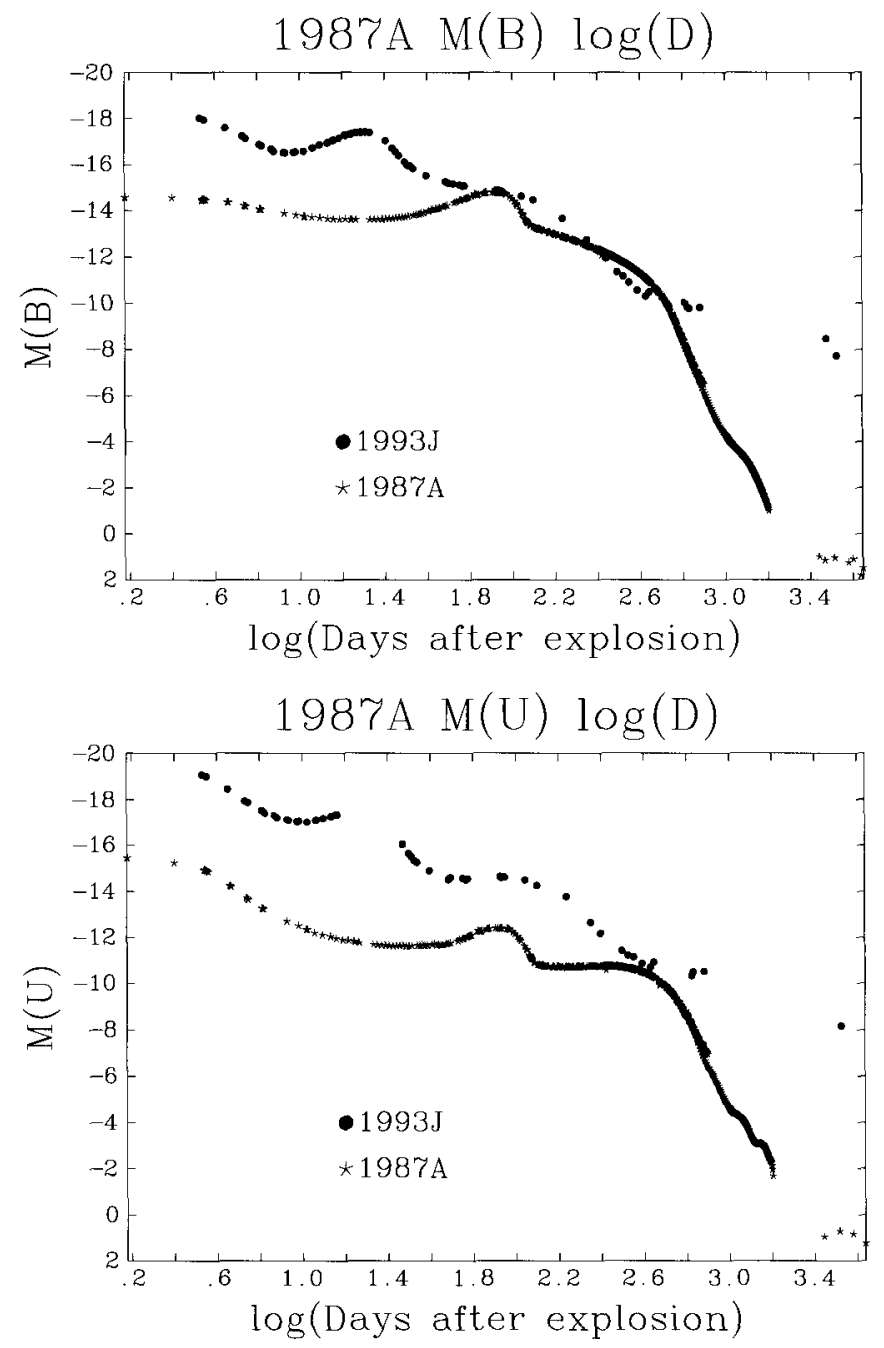

Fig. 1. Compilation of $U B$-band photometry for SN1993J and SN1987A. Note that at late times SN1993J seems to plateau out at very bright absolute magnitudes whereas SN1987A fades rapidly. The data come from mostly [2] (SN1987A) and [4] (SN1993J) with the points at $\log (D)>3.4$ from our measured magnitudes in HST archive images.

illustrate evidence for strong interaction of the ejecta with a dense circumstellar medium. Hence the plateau effect may just be due to the strong emission line flux from this interaction. However it could also be due to the fact that there is an underlying stellar component, for example a supergiant star with an absolute magnitude in the range $-6<M_{B}<-8$. 


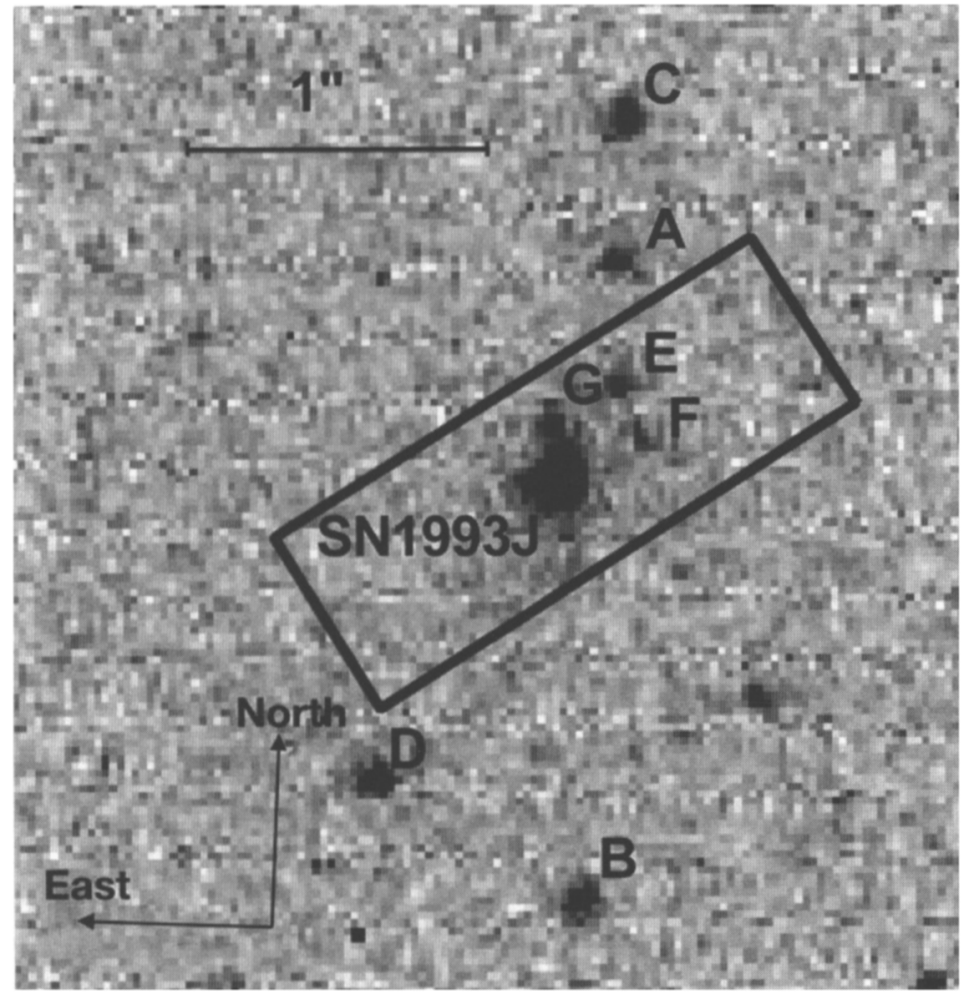

Fig. 2. HST ACS F330W image of SN1993J and its surrounding stellar population. This is a 1200s exposure taken 28 May 2002 (9.2yr after explosion). We follow the nomenclature of [8] and add an extra detected star in the ACS image (star G). The box is an example of the sky area sampled in the ground-based spectra from KeckI LRIS-B. The width is $0.7^{\prime \prime}$ (the slit-width) and length is $1.8^{\prime \prime}$ which is the aperture size used in the spectral reductions. The full photometry results are presented in $[5]$.

\section{Searching for the Companion Star}

To search for signs of a hot companion star at the position of $1993 \mathrm{~J}$ we took HST ACS images in the UV and deep, medium resolution spectra at Keck (full details are in [5]). Our idea was to take high signal-to-noise spectra in the near-UV to look for the spectral signature of an OB-supergiant or massive main-sequence star i.e. HI, HeI or HeI absorption features. The ACS image is shown on Fig. 2. SN1993J has several neighboring blue stars (G-F are at distances of 3-6 pc from the $\mathrm{SN}$ ), but there is no dense OB-association.

We took a $5.5 \mathrm{hr}$ spectrum with Keck using a $0.7^{\prime \prime}$ slit and we estimate that the ground-based spectrum samples the region within the box in Fig. 1 (the seeing was between $0.7-1.2^{\prime \prime}$ ). The spectrum is shown in Fig. 3. The 


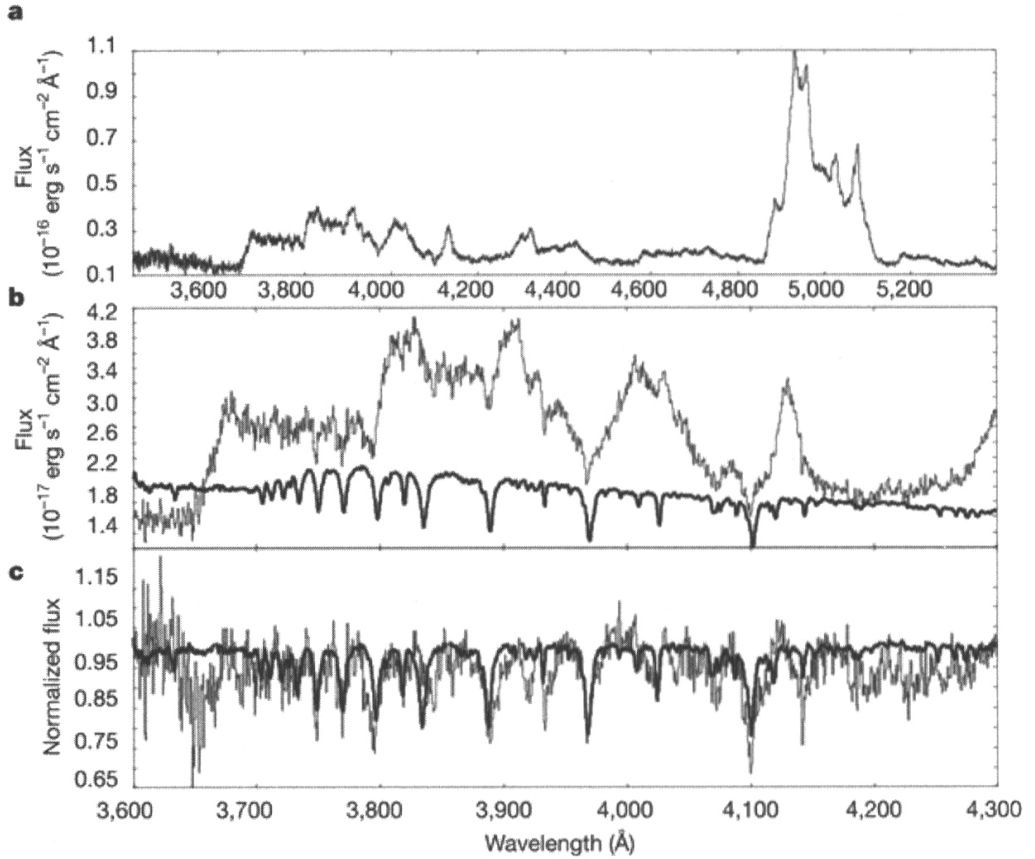

Fig. 3. Upper panel: flux calibrated spectrum of SN1993J 9.93yrs after explosion. Resolution 2.4A, S/N ratio between 15-30. Middle Panel: the near UV spectrum of SN1993J and a B1Ia supergiant spectrum (HD168489) which has been scaled to the distance of M81 with a reddening of $E(B-V)=0.2$ and a velocity shift of -120 $\mathrm{km} \mathrm{s}{ }^{-1}$ applied. The sharp absorption features seen on 1993J are coincident with the $\mathrm{HI}$ and HeI wavelengths. Bottom Panel: Normalized spectra of SN1993J and the B-type supergiant. The SN spectrum was normalized using short spline fits to attempt to remove the broad emission-line component and allow the stellar spectral contribution to be estimated. The absorption lines are best matched with an early type B-supergiant (B0-B4) with a B2Ia spectrum giving the most consistent fit to all constraints.

broad emission lines characteristic of the strong circumstellar interaction are clearly visible but there are some striking, narrow absorption features superimposed on the continuum of the supernova. These features are all coincident with $\mathrm{HI}$ or HeI lines in an OB-star. This spectrum is a composite between the emission flux from the SN ejecta interaction and flux from an OB-star or stars. Clearly the important issue is whether or not the flux from the surrounding stars we see in the ACS images can contribute enough to cause these lines. Using the ACS photometry, we simulated the effect of the surrounding stars using a Gaussian weighting function (weighting the contributions by the distance from the centroid of SN1993J in a similar manner to [8]) and determined the maximum contribution of the surrounding stars to the flux of the 
ground-based spectrum of SN1993J to be $18 \%$ [5]. We took some representative normalized spectra of OB-supergiants and diluted the continuum to $18 \%$. The calculated line strengths are much too weak to match the observed features in Fig. 3.

To match the strength of these lines we took B-type supergiant spectra, added excess continuum to the stellar spectra (simulating the effect of the SN flux contribution) and renormalized. We varied the flux ratio until the renormalized H I line strengths matched those observed in the SN1993J spectrum. In a similar manner we matched the He l lines at $3819 \AA$ and $4026 \AA$, assuming a normal He abundance. Early B-type supergiant spectra in the range B0-B4 provided the most consistent fit. Each line was fitted individually and the final ratios were calculated from the mean of all features. After accounting for the $18 \%$ contamination for the surrounding stars we estimate that the following continua ratios for each spectral type would give a consistent match $\mathrm{SN} / \mathrm{B} 0 \mathrm{Ia}=1, \mathrm{SN} / \mathrm{B} 1 \mathrm{Ia}=1.9, \mathrm{SN} / \mathrm{B} 2 \mathrm{Ia}=1.9, \mathrm{SN} / \mathrm{B} 3 \mathrm{Ia}=2.3, \mathrm{SN} / \mathrm{B} 4 \mathrm{Ia}=3$. The pre-explosion $U B$ photometry was used to further constrain the luminosity of the progenitor and our best estimate of a star that could produce the narrow lines and match the prediscovery photometry is $\log T_{\text {eff }}=4.3 \pm 0.1$ and $\log L / L_{\odot}=5 \pm 0.3$ (which is approximately B2Ia). Using these values and the prediscovery photometry of [1] we further restrict the progenitor to be a K-type supergiant with parameters $\log T_{\text {eff }}=3.63 \pm 0.05$ and $\log L / L_{\odot}=5.1 \pm 0.3$.

These results confirm that the progenitor of SN1993J was indeed a member of a binary system. A binary system of components with initial masses $14 \mathrm{M}_{\odot}$ and $15 \mathrm{M}_{\odot}$ is consistent with the observations. The $15 \mathrm{M}_{\odot}$ becomes a red-supergiant, loses almost all its envelope $\left(\sim 8 M_{\odot}\right)$ to its companion and then explodes when it has a mass of $\sim 5.4 M_{\odot}$ (and helium exhausted core of $\sim 5.1 M_{\odot}$ ). A full discussion is available in [5].

\section{References}

1. G. Aldering, R.M. Humphreys, M. Richmond: Astron. J. 107, 662 (1993)

2. J.A. Caldwell et al. : Mon. Not. R. Astron. Soc. 262, 313 (1993)

3. J.G. Cohen, J. Darling, A. Porter: Astron. J. 110, 308 (1995)

4. J. Lewis et al. : Mon. Not. R. Astron. Soc. 266, 27 (1994)

5. J.R. Maund, S.J. Smartt, R.P. Kudritzki, P. Podsiadlowski, G.F. Gilmore: Nature 427, 129 (2004)

6. K. Nomoto et al. : Nature 364, 507 (1993)

7. P. Podsiadlowski, J.J.L. Hsu, P.C. Joss, R.R. Ross: Nature 364, 509 (1993)

8. S.D. Van Dyk et al. : Pub. Astron. Soc. Pacific 114, 1322 (2003)

9. S.E. Woosley, R.G. Eastman, T.A. Weaver, P.A. Pinto: Astrophys. J. 429, 300 (1994) 